\title{
Complexity and uncomplexity during energy injection
}

\author{
Mahsa Lezgi ${ }^{*}$ and Mohammad Ali-Akbari ${ }^{\dagger}$ \\ Department of Physics, Shahid Beheshti University, 1983969411 Tehran, Iran
}

(Received 1 April 2021; accepted 1 June 2021; published 24 June 2021)

\begin{abstract}
We consider a strongly coupled field theory with a critical point and a nonzero chemical potential at a finite temperature, which is dual to an asymptotically anti-de Sitter charged black hole. We study the evolution of the rescaled holographic subregion complexity near to and far from the critical point. We explain two distinct concepts of complexity in this theory and discuss that the state under study is complex based on how much information is needed to specify the state and is simple according to how many operations have to be done to reach the state. We justify the decreasing of the rescaled holographic subregion complexity with time using an increasing number of microstates of the mixed state and speculate about the description of the relative rescaled holographic subregion complexity of the initial state and the final state as a resource. We propose that in this process the complexity of the mixed state decreases and the complexity of the environment increases. We also observe that in this model the rescaled holographic subregion complexity is a good observable for probing the dynamical critical exponent.
\end{abstract}

DOI: 10.1103/PhysRevD.103.126024

\section{INTRODUCTION}

The evolution of out-of-equilibrium systems towards equilibrium is one of the most important areas of physics to study. If these systems are far-from-equilibrium and strongly coupled, they are much more complicated to investigate. By mapping strongly coupled field theories to weakly coupled gravity, gauge-gravity duality, or more generally the holographic idea, provides a useful framework to study different nonperturbative problems, including far-from-equilibrium phenomena [1,2]. Quark-gluon plasma produced in heavy ion collisions at the Relativistic Heavy Ion Collider is a good example of such a strongly coupled and far-from-equilibrium medium. Experimental observations show that the time scale that is needed for this plasma to reach thermal equilibrium is significantly shorter than the one that is expected from perturbative methods [3]. Holographic thermalization proposes that such a thermalization process is dual to the process of the formation of a black hole in the bulk, for instance the collapse of a thin-shell matter described by an anti-de Sitter-Vaidya (Vaidya-AdS) metric [4]. A far-from-equilibrium state in field theory can be prepared by the injection of energy using an external source and the time evolution of this

\footnotetext{
s_lezgi@sbu.ac.ir

m_aliakbari@sbu.ac.ir
}

Published by the American Physical Society under the terms of the Creative Commons Attribution 4.0 International license. Further distribution of this work must maintain attribution to the author(s) and the published article's title, journal citation, and DOI. Funded by SCOAP ${ }^{3}$. far-from-equilibrium system can be probed by local and nonlocal observables.

According to the holographic idea, a connection has been developed between quantities in quantum information theory and certain geometric quantities in the bulk geometry. For example, the Hubney-Ryu-Takayanagi proposal for entanglement entropy as a measure of quantum correlation of a pure quantum state is a simple geometric prescription that passes many tests successfully $[5,6]$. Holographic entanglement entropy can be used to classify various phase transitions and critical points $[7,8]$.

Complexity is another main concept in quantum information theory. The complexity of a state is defined as the minimum number of simple gates needed to produce it from some reference state [9]. In the context of quantum field theory, complexity refers to the minimum number of unitary operators needed to prepare a target state from a reference state [10]. In other words, complexity can classify various quantum states based on the difficulty of their creation. There exists two holographic prescriptions, namely the $\mathrm{CV}$ (complexity = volume) conjecture and the CA (complexity $=$ action) conjecture $[11,12]$. These proposals have been introduced for the complexity of a pure state in the whole boundary system and they both can extend to be defined on subregions corresponding to the complexity of mixed states $[13,14]$. Inspired by the Hubney-RyuTakayanagi proposal, the complexity for a subsystem on the boundary is equal to the volume of the codimensional-one hypersurface enclosed by the Hubney-Ryu-Takayanagi surface, which is known as holographic subregion complexity (HSC) [14]. Some works on CV and CA conjectures and on HSC for various gravity models can be found in [15-24]. 
Complexity is like the entropy of an auxiliary classical system, which describes the evolution of operator $U(t)$ on $S U\left(2^{k}\right)$ [25]. This description opens the window to a topic known as the thermodynamics of complexity. For example, the second law of complexity is just the usual second law of thermodynamics applied to the auxiliary classical system. Moreover, in the thermodynamical view the lack of entropy or the difference between the maximum entropy and the actual entropy, i.e., negentropy, is a resource for doing a work. There exists a concept analogous to negentropy in the thermodynamics of complexity. This parallel definition of the resource for complexity, called uncomplexity, is the difference between the maximum possible complexity and the actual complexity. In this case, work refers to doing directed computation, meaning a computation with a goal which is called computational work $[25,26]$.

In this paper we investigate the evolution of HSC on a far-from-equilibrium state, dual to a Vaidya charged black hole, in the presence of temperature and chemical potential. We consider a gravity background dual to a field theory with a critical point and study the behavior of the relaxation time and the HSC as the system moves towards the critical point. The questions we are interested in are: Is HSC a proper observable to probe the dynamical critical exponent? Does the existence of a critical point in the theory help to distinguish between the two concepts of complexity for determining whether a state is complex or simple? Considering a black hole as a computational machine, can a resource be defined for it in this time evolution? We start by introducing the background and then compute the HSC on the background and discuss its behavior to answer the above questions.

\section{REVIEW ON THE BACKGROUND}

A critical point can be described as a point at which the line of the first-order phase transition must end. Field theory observables can be studied near the critical point and one can also compute the associated (dynamical) critical exponent. Therefore, here we review a charged black hole solution which is holographically dual to a strongly coupled field theory with a critical point. The metric we are interested in, called charged dilaton anti-de Sitter (AdS) [27], is

$$
\begin{aligned}
d s^{2}= & -N(z) f(z) d t^{2}+\frac{d z^{2}}{z^{4}\left(1+b^{2} z^{2}\right) f(z)} \\
& +\frac{1+b^{2} z^{2}}{z^{2}} g(z) d \vec{x}^{2},
\end{aligned}
$$

where $z$ is the radial coordinate, $\vec{x} \equiv\left(x_{1}, x_{2}, x_{3}\right)$, and

$$
\begin{aligned}
& f(z)=\frac{1+b^{2} z^{2}}{z^{2}} \Gamma^{2 \gamma}-\frac{m z^{2}}{1+b^{2} z^{2}} \Gamma^{1-\gamma}, \\
& N(z)=\Gamma^{-\gamma}, \quad g(z)=\Gamma^{\gamma}, \quad \Gamma(z)=1-\frac{b^{2} z^{2}}{1+b^{2} z^{2}}, \quad \gamma=\frac{\alpha^{2}}{2+\alpha^{2}} .
\end{aligned}
$$

$\alpha$ determines the coupling constant between the gauge field and the dilaton. The constant $b$ is related to $m$, the mass of the black hole and $q$, the charge of the black hole, as follows:

$$
q=\sqrt{\frac{6 m}{2+\alpha^{2}}} b .
$$

This background is asymptotically $\mathrm{AdS}_{5}$. The field theory lives on the boundary where it is located at $z=0$. The Hawking temperature of the black hole, corresponding to the temperature of the field theory, is

$$
T=\frac{b \Gamma\left(z_{h}\right)^{\frac{3 \gamma}{2}-1}}{4 \pi \sqrt{1-\Gamma\left(z_{h}\right)}}\left(2(3 \gamma-1)-3(2 \gamma-2) \Gamma\left(z_{h}\right)\right),
$$

where $z_{h}$ is the horizon. Due to the gauge field in the balk, the chemical potential in the field theory is [27]

$$
\mu=\frac{b \sqrt{3 m}}{\sqrt{2\left(\alpha^{2}+2\right)\left(b^{2}+\frac{1}{z_{h}^{2}}\right)}} .
$$

In the case of $\alpha=2$, the above background is identical with the one that enjoys a critical point at $\left(\frac{\mu}{T}\right)_{*}=1.1107$ and its phase diagram can be found in [28]. To be more precise, using (4) and (5) one easily obtains

$$
b z_{h}=\frac{1 \pm \sqrt{1-\frac{8 \mu^{2}}{\pi^{2} T^{2}}}}{\frac{2 \mu}{\pi T}},
$$

where

$$
z_{h}=\sqrt{\frac{b^{2}+\sqrt{b^{4}+4 m}}{2 m}} .
$$

Therefore, for each value of $\frac{\mu}{T}$, there are two distinct values of $b z_{h}$ which specify stable and unstable branches of solutions indicating that there exists a phase transition in field theory and the critical point is where the two branches merge [28]. The upper (lower) sign in (6) corresponds to thermodynamically unstable (stable) solutions. For a thermodynamically stable solution the Jacobian, $\mathcal{J}=\frac{\partial(s, \rho)}{\partial(T, \mu)}$, is positive [29]. $s$ and $\rho$ are entropy and charge density, respectively, and 


$$
\begin{gathered}
s \propto \frac{T^{3}\left(1+b^{2} z_{h}^{2}\right)^{2}}{\left(2+b^{2} z_{h}^{2}\right)^{3}}, \\
\rho \propto \frac{\mu}{T}\left(2+b^{2} z_{h}^{2}\right) \sqrt{1+b^{2} z_{h}^{2}} .
\end{gathered}
$$

Note that with some field redefinition this is the holographic model of the thermal QCD critical point [29], in which temperature is proportional to the parameter $b$.

Calculating the relaxation time and the investigation of the time evolution of HSC are of interest in this paper. Thus, we consider a Vaidya-type solution found by replacing $f(z)$ with $f(z, v)$, in which

$$
d v=d t-\frac{d z}{z^{2} \sqrt{\left(1+b^{2} z^{2}\right) N(z)} f(z)} .
$$

Note that $t$ and $v$ are equal at the boundary. This replacement has been done by exchanging $m$ and $q$ with $m I(v)$ and $q \sqrt{I(v)}$, respectively. $I(v)$ is the timedependent function which is chosen to be

$$
I(v)=\frac{1}{2}\left(1+\tanh \left(\frac{v}{v_{0}}\right)\right),
$$

where $v_{0}$ indicates the speed of energy injection into the background. In fact, it measures how fast the function can reach a maximum value. In the literature, $v_{0} \ll 1\left(v_{0} \gg 1\right)$ is called fast (slow) quench. Different functions, which can be chosen instead of (11), have been discussed in [30,31], and it seems that the results are independent of the picked functions. By replacing the above equations in (1), the Vaidya-AdS-like (VAdS) solution is

$$
\begin{aligned}
d s^{2}= & -N(z) f(z, v) d v^{2}-\frac{2}{z^{2}} \sqrt{\frac{N(z)}{1+b^{2} z^{2}}} d v d z \\
& +\frac{1+b^{2} z^{2}}{z^{2}} g(z) d \vec{x}^{2} .
\end{aligned}
$$

We use the VAdS metric to discuss the time evolution of the HSC.

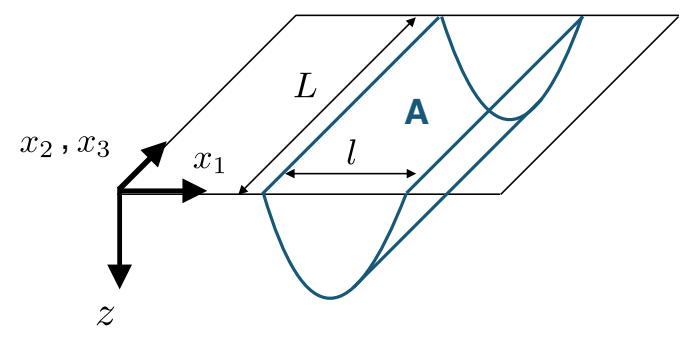

FIG. 1. A strip entangling surface of length $l$ and width $L \rightarrow \infty$ in a static AdS background.

\section{HOLOGRAPHIC SUBREGION COMPLEXITY}

The complexity of the mixed state (the subregion $A$ on the boundary), motivated by the Hubney-Ryu-Takayanagi proposal, is dual to the volume enclosed by the extremal surface $\gamma_{A}$ appearing in the computation of holographic entanglement entropy [14], i.e.,

$$
\mathcal{C}_{A}=\frac{V_{\gamma_{A}}}{8 \pi R G_{N}}
$$

where $R$ and $G_{N}$ are the AdS radius and the Newton constant, respectively. $\mathcal{C}_{A}$ is the HSC for the subregion $A$. In order to compute $\gamma_{A}$ at a given time, we consider the subsystem $A$ which is defined as

$$
-\frac{l}{2}<x_{1}(\equiv x)<\frac{l}{2}, \quad x_{2} \in(-\infty,+\infty), \quad x_{3} \in(-\infty,+\infty),
$$

and describes a strip surface of length $l$ and width $L \rightarrow \infty$, as you can see in Fig. 1 in a static AdS background. For a dynamical background, $\gamma_{A}$ does not live on a constant time slice. Duo to the symmetry of the strip, the external surface $\gamma_{A}$ can be parametrized as

$v=v(x), \quad z=z(x), \quad v\left(\frac{l}{2}\right)=t, \quad z\left(\frac{l}{2}\right)=z_{0}$,

where $z_{0}$ is a UV cutoff. Then the area of the minimal surface turns out to be

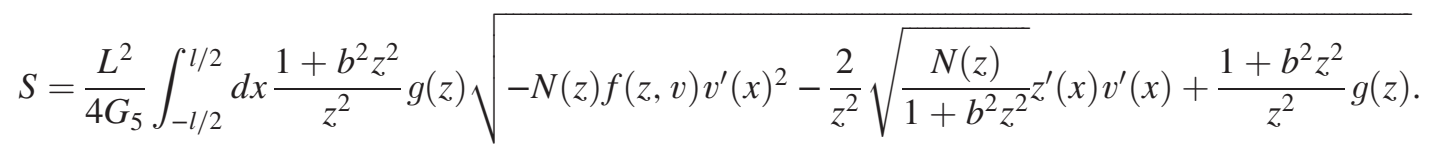

Considering the integrand in (16) as a Lagrangian, where the symmetry of the problem indicates that the turning point of the minimal surface lies at $x_{1}=0$, we therefore used

$$
v^{\prime}(0)=z^{\prime}(0)=0, \quad v(0)=v_{*}, \quad z(0)=z_{*} .
$$

One can find the equations of motion for $z(x)$ and $v(x)$ and then, using (17), they can be numerically solved to obtain the profiles of $z(x)$ and $v(x)$. In the case at hand, the volume can be parametrized by $v=v(x)$ and $z=z(x)$, or equivalently $z=z(v)$. For the background solution (12), the induced metric on the volume is 


$$
d s^{2}=-\left(N(z) f(z, v)+\frac{2}{z^{2}} \sqrt{\frac{N(z)}{1+b^{2} z^{2}} \frac{\partial z}{\partial v}}\right) d v^{2}+\frac{1+b^{2} z^{2}}{z^{2}} g(z)\left(d x^{2}+\sum_{i=1}^{2} d x_{i}^{2}\right)
$$

and the volume becomes

$$
V=2 L^{2} \int_{v_{*}}^{v\left(\frac{l}{2}\right)} d v \int_{0}^{x(v)} d x \frac{1+b^{2} z^{2}}{z^{2}} g(z) \sqrt{\frac{1+b^{2} z^{2}}{z^{2}} g(z)\left(-N(z) f(z, v)-\frac{2}{z^{2}} \sqrt{\frac{N(z)}{1+b^{2} z^{2}} \frac{\partial z}{\partial v}}\right)} .
$$

Clearly in the above equation $\int_{0}^{x(v)} d x$ can be replaced by $x(v)$. Since the HSC (or its equivalent volume in the dual gravity) is divergent, we would like to introduce a normalized version of the HSC using (13), as follows:

$$
C \equiv \frac{8 \pi R G_{N}\left(\mathcal{C}_{V \text { AdS }}-\mathcal{C}_{\mathrm{AdS}}\right)}{L^{2}}=\frac{V-V_{\mathrm{AdS}}}{L^{2}}
$$

where $\mathcal{C}_{V \text { AdS }}$ and $\mathcal{C}_{\text {AdS }}$ are the HSCs for $A$ in VAdS and AdS geometry, respectively. The volumes are defined for the same boundary region such that $V$ in Eq. (19) reduces to $V_{\text {AdS }}$ by setting $b$ and $m$ equal to zero.

In order to find the relaxation time scale for the complexity, we introduce the following function:

$$
\epsilon(t)=\left|1-\frac{C(t)}{C(\infty)}\right|
$$

Then the relaxation time for the $\mathrm{HSC}, t_{c}$, is the time at which $\epsilon(t)<10^{-3}$ and it stays below this limit forever. We will numerically calculate the time scale $t_{c}$ later on.

\section{NUMERICAL RESULTS}

In this section we will present our results from the numerical calculation of the HSC at the VAdS metric (12). We will study the evolution of the HSC for

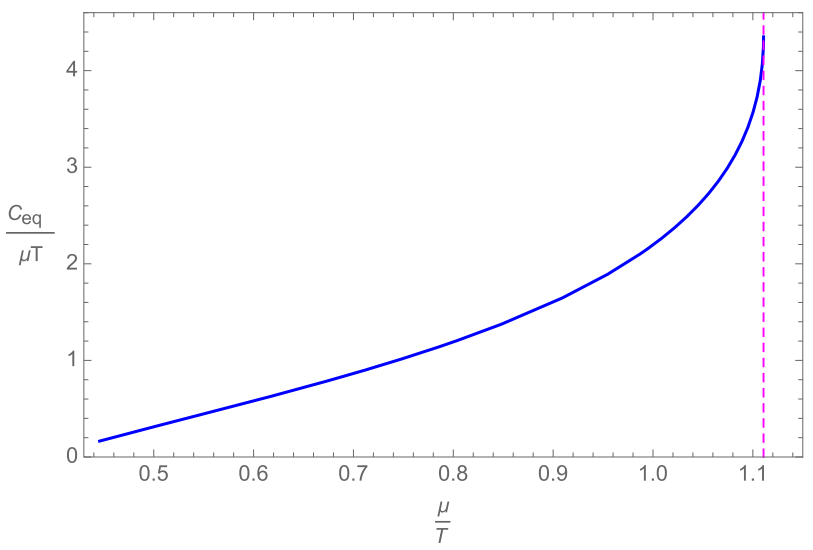

an out-of-equilibrium process corresponding to the VAdS background in the gravity dual.

We are interested in investigating the behavior of the HSC as we move towards the critical point, $\left(\frac{\mu}{T}\right)_{*}$, in the field theory. In Fig. 2, we have plotted the final value of the rescaled HSC (RHSC), $\frac{C_{e q}}{\mu T}$, meaning $\frac{C}{\mu T}$ at $t \rightarrow \infty$, and the rescaled relaxation time, $v_{0}^{-1} t_{c}$, as a function of $\frac{\mu}{T}$. In the left panel, $\frac{C_{e q}}{\mu T}$ and $\frac{\mu}{T}$ increase together. By increasing $\frac{\mu}{T}$, the amount of information it takes to specify the final state increases. In the right panel, we can observe how with increasing $\frac{\mu}{T}$ the time it takes for the RHSC to relax decreases. This interesting result means that by increasing $\frac{\mu}{T}$, although the final value of the RHSC increases, it takes less time to get the RHSC equilibrium.

It seems that moving towards the critical point can highlight the differences between the two concepts of complexity; that is, how much information it takes to specify a state and how many operations need to be done to reach the state. Using the terms prevalent in quantum or classical information literatures, we can consider the space of states described by $\mathrm{K}$ classical bits $(010111001 \ldots)$. Our desired task is to start from a state in which all bits are the same and reach the state in which the bits alternate. This target state $(010101010 \ldots)$ can be complex from one point of view and simple from another. How many simple operations have to be done to

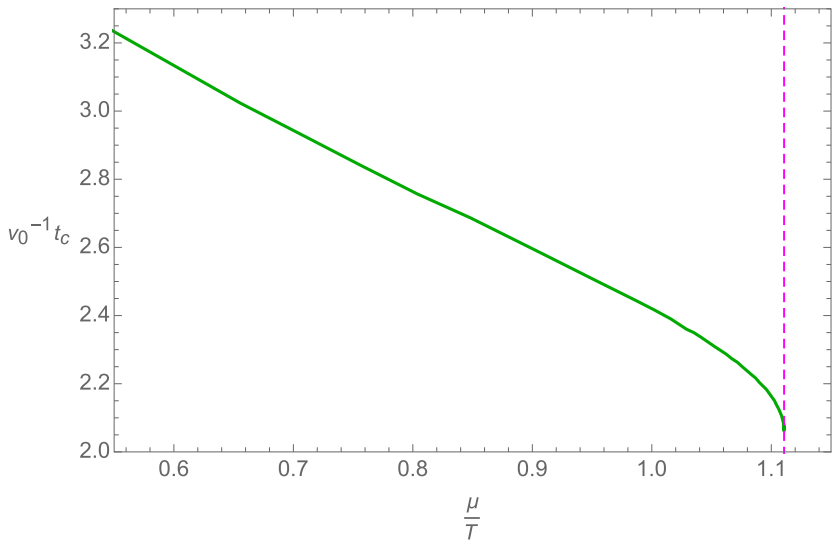

FIG. 2. Left: The behavior of the final value of the RHSC, $\left.\frac{C}{\mu T}\right|_{t \rightarrow \infty}=\frac{C_{e q}}{\mu T}$, as a function of $\frac{\mu}{T}$ for $l=0.3$ and $v_{0}=3$. The magenta dashed line indicates the critical point which is at $\left(\frac{\mu}{T}\right)_{*}$. Right: The behavior of the rescaled relaxation time, $v_{0}^{-1} t_{c}$, with respect to $\frac{\mu}{T}$. 

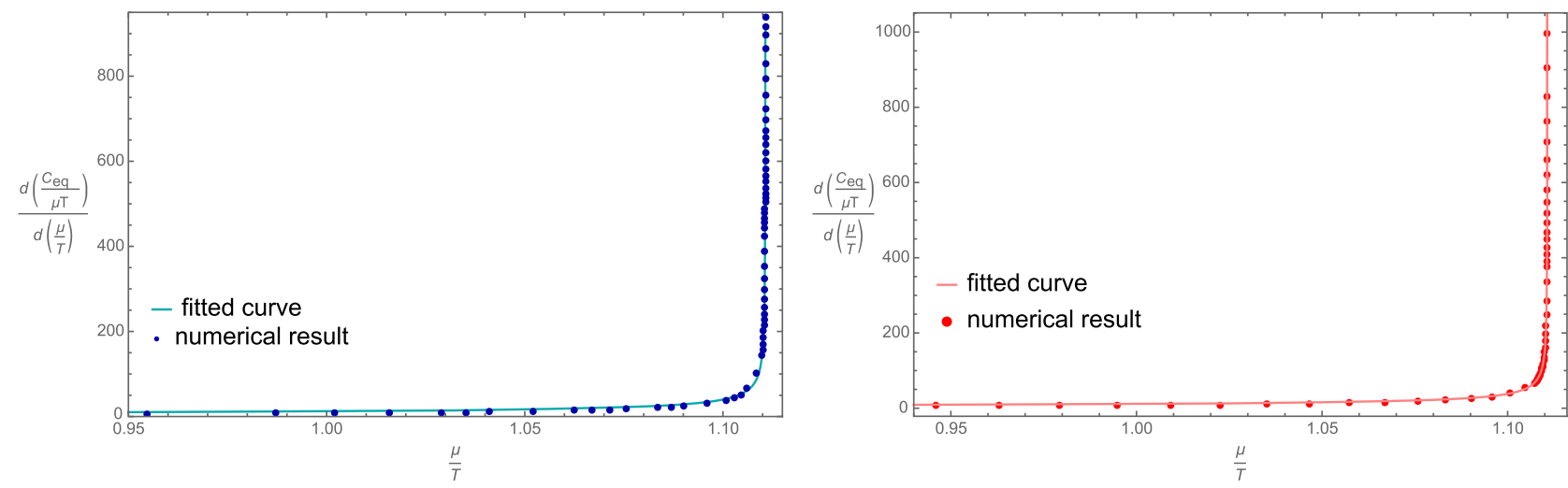

FIG. 3. Left: The slope of the final value of the RHSC, $\frac{C_{e q}}{\mu T}$, as a function of $\frac{\mu}{T}$ for $l=0.3$ and $v_{0}=3$, near the critical point. The light blue curve is the function $\left(\frac{\pi}{2 \sqrt{2}}-\frac{\mu}{T}\right)^{-\theta}$ fitted with the data by $\theta=0.501662$. Right: The slope of $\frac{C_{e q}}{\mu T}$ as a function of $\frac{\mu}{T}$ for $l=0.3$ and $v_{0}=0.05$. The pink curve is fitted with the data by $\theta=0.513942$.

reach the target state, or how long it has to run, does depend on the number of bits and thus $(010101010 \ldots)$ is complex from this aspect. It is simple if we consider how much information is needed to specify the target state, since it does not depend on the number of bits [32]. It seems that we observe such behavior in the case that we study. On one hand the final value of the RHSC increases due to the amount of information required to specify the final state by increasing $\frac{\mu}{T}$, and on the other hand, based on the time it takes to run, the final state or the task that has to be done for getting to the final state is simple. We speculate this decrease of the time it takes to reach the final state, or the simplicity of the final state from this point of view, could be because of movement to the critical point of the theory, since near the critical point universality classes are characterized with a large scale behavior and the theory can be described with fewer parameters.

An interesting question is whether the HSC understands about the phase structure of the theory. As you can see in Fig. 2, the slope of $\frac{C_{e q}}{\mu T}$ approaches infinity at $\left(\frac{\mu}{T}\right)_{*}$. We can define

$$
\frac{d\left(\frac{C_{e q}}{\mu T}\right)}{d \frac{\mu}{T}}(i)=\frac{\frac{C_{e q}}{\mu T}(i+1)-\frac{C_{e q}}{\mu T}(i)}{\frac{\mu}{T}(i+1)-\frac{\mu}{T}(i)},
$$

where $i$ indicates the $i$ th point of data points. The slope of the curve for the points near the critical point can be fitted with a function of the form $\left(\frac{\pi}{2 \sqrt{2}}-\frac{\mu}{T}\right)^{-\theta}$, where $\theta$ is the dynamical critical exponent [28]. In Fig. 3, we have plotted the slope (22) near the critical point. We get $\theta=0.50166$ and $\theta=0.513942$ for slow and fast quench, respectively, which are in perfect agreement with the one that is obtained from the behavior of scalar quasi-normal modes [29]. Hence, HSC is a good observable for probing the dynamical critical exponent. Various observables have been studied to find the critical exponent [33-35].

In Fig. 4, on the left, we have plotted the RHSC as a function of rescaled time for two different values of $v_{0}$. At slow quench, $v_{0}=3$, the system has enough time to equilibrate during the transition and undergoes an adiabatic evolution. However, at fast quench, $v_{0}=0.05$, with a rapid altering, the RHSC experiences an increase at the early stage that reaches a maximum value and then decreases to a
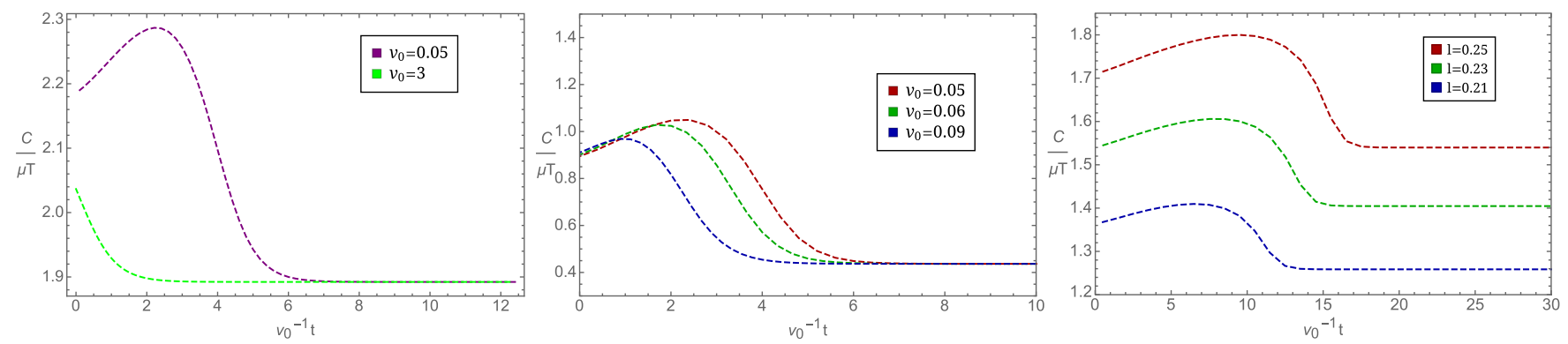

FIG. 4. Left: RHSC, $\frac{C}{\mu T}$, as a function of $v_{0}^{-1} t$ for $l=0.3$ and two values of $v_{0}$ at fixed $\frac{\mu}{T}=0.954716$. Middle: RHSC in terms of rescaled time for three values of $v_{0}$ at fixed $\frac{\mu}{T}=0.546466$ for $l=0.3$. Right: RHSC in terms of rescaled time for three values of $l$ at fixed $\frac{\mu}{T}=0.94597$ for $v_{0}=0.01$. 
constant value at a late time, $C_{e q}$. The faster the energy injection, the sooner the quench happens, and the earlier the system reaches complexity equilibrium. A different manner of energy injection into the system leads to different responses during the early time interval. Therefore, if we know only about the $T$ and $\mu$ in the boundary field theory, RHSC can distinguish between fast or slow quench. To study the effects of $v_{0}$ on the evolution of the RHSC, we have plotted RHSC as a function of rescaled time in the middle panel of Fig. 4. With the increase of $v_{0}$, in the range of fast quench, the maximum value which RHSC can reach becomes smaller and is achieved sooner. The evolution of the RHSC for the three values of $l$ has been shown in the right panel of Fig. 4. With the decrease of $l$ the RHSC decreases and reaches $C_{e q}$ sooner.

Holographic entanglement entropy calculation can be found in [27] for this background. The relation between holographic entanglement entropy and HSC for a mixed state has been widely discussed before [36-39]. In our case, no newer results were found. Contrary to the behavior of the HSC, during the quench, holographic entanglement entropy increases at the first stage and then arrives to a constant maximum value at a later time, but both of them reach a constant final value at $t \gtrsim \frac{l}{2}$.

Due to the second law of quantum complexity, a process with decreasing complexity is counterintuitive in the boundary field theory from the thermodynamical point of view [26]. We would like to claim that this second law of total quantum complexity also applies to the subregion complexity case. In comparison with holographic entanglement entropy, for a general Vaidya-AdS solution it is a monotonically increasing function of time compatible with the second law of the thermal entropy [5]. However, there may be cases where holographic entanglement entropy will decrease after a small perturbation due to the decoherence phenomenon [5]. Note that the decoherence phenomenon is related to the loss of information from a system into the environment and the effect of sharing quantum information with the surroundings [40]. According to our observations about the behavior of the RHSC of the state under study, we claim that the second law of complexity holds for our case similarly to the case of holographic entanglement entropy, and it will be justified more in the following. This behavior of HSC in these VaidyaAdS-like geometries has been reported in [36-39] and an attempt has been made to interpret it qualitatively using interpretation of $\mathrm{HSC}$ as a purification complexity [39]. What we want to emphasize are as follows:

(i) As shown in Fig. 4 in the left, middle, and right panels, in the evolution from the initial state corresponding to the VAdS background in initial time (which is a thermal state with a temperature proportional to the parameter $b$ ), to the thermal state corresponding to the VAdS background in final time, the RHSC decreases. In the initial RHSC, $C_{0}$ is always bigger than in the final RHSC, $C_{e q}$.
According to quantum statistical mechanics, there is an ensemble of microstates corresponding to a given mixed macrostate. At zero temperature corresponding to the zero entropy, there exists one microstate, which is called unique configuration, but nearing the equilibrium with increasing temperature, the number of these microstates increases. In order to specify the macrostate, it is not necessary to know the details of the underlying system. As in statistical systems, for instance, the speed of sound is determined only with the macroscopic characteristics of the system, i.e., pressure and density. In our case, to give an effective description of the mixed macrostate we do not need the details of the microstates that lead to it. Therefore, in the time evolution of the mixed state that we are studying in this paper, with the increase of temperature and as we near the equilibrium, we specify the state with less information than we do the state in initial time, which corresponded to the smaller number of microstates. Since the underlying field theory is conformal, our results are $\frac{\mu}{T}$ dependent, i.e., increasing chemical potential, in this case, is like decreasing temperature, and vice versa. Hence, this explanation is in agreement with the left panel of Fig. 2.

(ii) Decreasing complexity from its maximum possible value or lack of complexity is a resource for a computational work $[25,26]$. In analogy with thermodynamic free energy that is used to do a work, uncomplexity is a resource for doing a directed computation [26]. Therefore a quantum computer which has been run and has reached maximum complexity is useless for doing any computations. With the decrease of complexity of the black hole as a computational machine, we have a resource for performing a directed quantum computation. We can interpret the relative RHSC of the initial state and the final state as a resource which is expended to reach the final state. We call it as follows:

$$
\Delta C \equiv C_{e q}-C_{0} .
$$

In the left panel of Fig. 5, the RHSC as a function of rescaled time for three values of $\frac{\mu}{T}$ have been plotted. As we near the critical point, $C_{0}$ and $C_{e q}$ increase and $C_{e q}$ is smaller than $C_{0}$. In the middle and right panel of Fig. 5, $\Delta C$ as a function of $\frac{\mu}{T}$ has been plotted for fast and slow quench. Nearing the critical point, $\Delta C$ decreases and at the critical point, the magenta dashed line, the slope of the curve approaches infinity. It seems that with the decrease of the needed resource for reaching the final state, $\Delta C$, as it approaches the critical point, the time it takes to expend the resource decreases too, in agreement with the right panel of Fig. 2. 

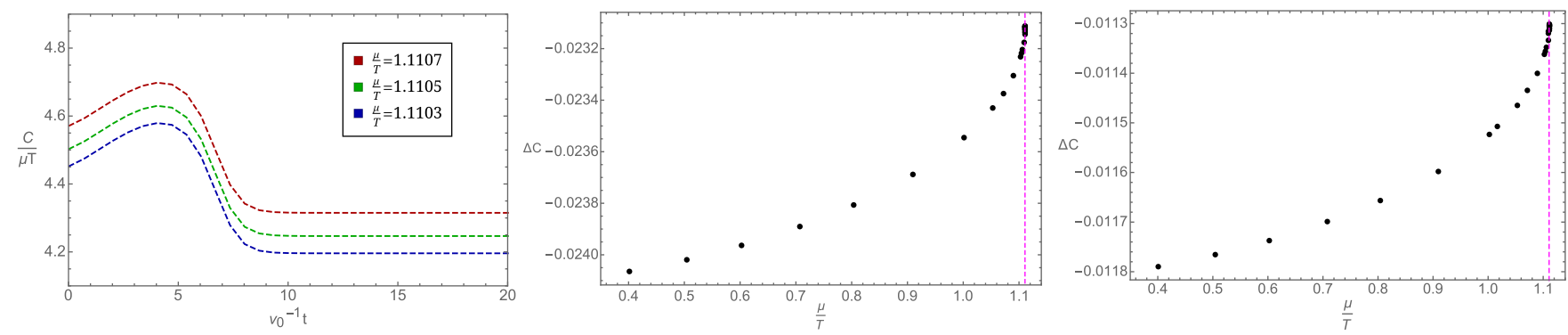

FIG. 5. Left: RHSC, $\frac{C}{\mu T}$, as a function of $v_{0}^{-1} t$ for three different values of $\frac{\mu}{T}, l=0.3$, and $v_{0}=0.05$. Middle: $\Delta C$ in terms of $\frac{\mu}{T}$ for $v_{0}=0.05$ and $l=0.3$. The magenta dashed line shows the critical point. Right: $\Delta C$ in terms of $\frac{\mu}{T}$ for $v_{0}=3$ and $l=0.3$.
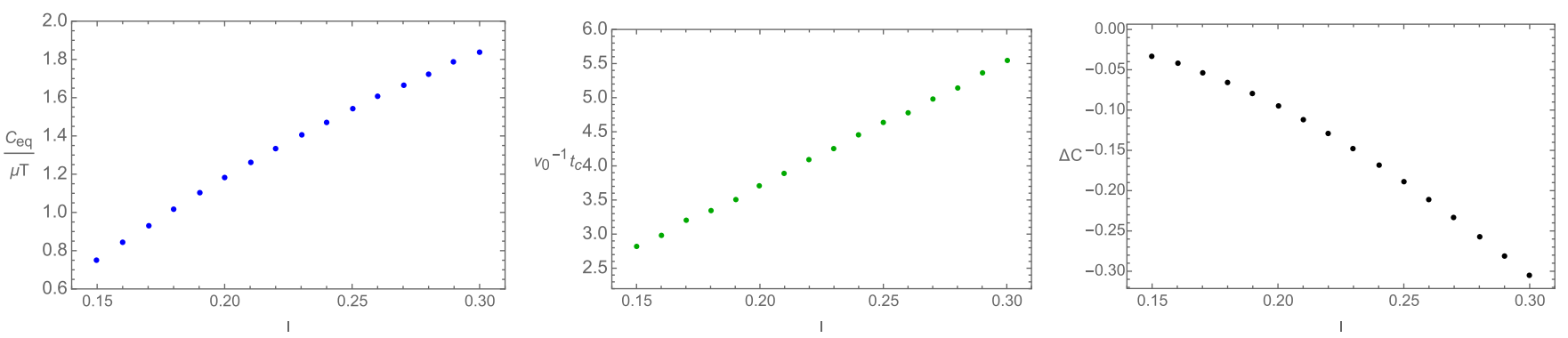

FIG. 6. In all three panels $\mu=0.274601, T=0.290286$, and $v_{0}=0.07$. Left: The final value of the RHSC, $\frac{C_{e q}}{\mu T}$, as a function of $l$. Middle: The behavior of the rescaled relaxation time, $v_{0}^{-1} t_{c}$, with respect to $l$. Right: $\Delta C$ in terms of $l$.

(iii) The system we study consists of a state and its environment. In this particular case, the external source that injects energy into the boundary field theory is the environment. In initial time the external source is zero and thus its complexity, or the information for specifying it, is zero too. It is obvious during the evolution when the external source value becomes nonzero, as we need the information to specify it. Therefore, in this evolution, the complexity of the external source increases. In contrast, the effect of the external source on the state, as you can see in the left panel of Fig. 5, is the decrease of complexity. We speculate that in this process, the complexity of the environment increases and the complexity of the state decreases. Therefore, it seems the second law of complexity is not violated for the whole system. (iv) In the left panel of Fig. 6, the final value of the RHSC as a function of length of the subregion for fixed $\mu$ and $T$ has been plotted. $\frac{C_{e q}}{\mu T}$ and $l$ increase together which means that by increasing $l$ the amount of information for specifying the final state increases. In the middle of Fig. 6, it has been shown that the time it takes for the RHSC to relax increases with the increase of $l$. In the right panel of Fig. 6, $\Delta C$ as a function of $l$ shows that by increasing $l, \Delta C$ or the resource increases too. Therefore, in agreement with our previous results, it seems that with the increase of the needed resource for reaching the final state by increasing $l$, the time it takes to expend the resource to reach the final state increases too, as shown in the middle of Fig. 6.
[1] J. Casalderrey-Solana, H. Liu, D. Mateos, K. Rajagopal, and U. A. Wiedemann, Gauge/String Duality, Hot QCD and Heavy Ion Collisions (Cambridge University Press, Cambridge, England, 2014).

[2] V.E. Hubeny and M. Rangamani, A holographic view on physics out of equilibrium, Adv. High Energy Phys. 2010, 1 (2010).
[3] R. Baier, A. H. Mueller, D. Schiff, and D. T. Son, Bottom up thermalization in heavy ion collisions, Phys. Lett. B502, 51 (2001).

[4] V. Balasubramanian, A. Bernamonti, J. de Boer, N. Copland, B. Craps. E. Keski-Vakkuri, B. Muller, A. Schafer, M. Shigemori, and W. Staessens, Thermalization of Strongly Coupled Field Theories, Phys. Rev. Lett. 106, 191601 (2011). 
[5] T. Nishioka, S. Ryu, and T. Takayanagi, Holographic entanglement entropy: An overview, J. Phys. A 42, 504008 (2009).

[6] M. Rangamani and T. Takayanagi, Holographic entanglement entropy, Lect. Notes Phys. 931, 1 (2017).

[7] I. R. Klebanov, D. Kutasov, and A. Murugan, Entanglement as a probe of confinement, Nucl. Phys. B796, 274 (2008).

[8] G. Vidal, J. I. Latorre, E. Rico, and A. Kitaev, Entanglement in Quantum Critical Phenomena, Phys. Rev. Lett. 90, 227902 (2003).

[9] J. Watrous, Quantum computational complexity, arXiv: 0804.3401.

[10] R. Jefferson and R. C. Myers Circuit complexity in quantum field theory, J. High Energy Phys. 10 (2017) 107.

[11] D. Stanford and L. Susskind, Complexity and shock wave geometries, Phys. Rev. D 90, 126007 (2014).

[12] A. R. Brown, D. A. Roberts, L. Susskind, B. Swingle, and Y. Zhao, Complexity, action, and black holes, Phys. Rev. D 93, 086006 (2016).

[13] D. Carmi, R. C. Myers, and P. Rath, Comments on holographic complexity, J. High Energy Phys. 03 (2017) 118.

[14] M. Alishahiha, Holographic complexity, Phys. Rev. D 92 , 126009 (2015).

[15] O. Ben-Ami and D. Carmi, On volumes of subregions in holography and complexity, J. High Energy Phys. 11 (2016) 129.

[16] S. J. Zhang, Complexity and phase transitions in a holographic QCD model, Nucl. Phys. B929, 243 (2018).

[17] S. J. Zhang, Subregion complexity in holographic thermalization with dS boundary, Eur. Phys. J. C 79, 715 (2019).

[18] P. Roy and T. Sarkar, On subregion holographic complexity and renormalization group flows, Phys. Rev. D 97, 086018 (2018).

[19] R. Fareghbal and P. Karimi, Complexity growth in flat spacetimes, Phys. Rev. D 98, 046003 (2018).

[20] M. Alishahiha, A. F. Astaneh, M. R. M. Mozaffar, and A. Mollabashi, Complexity growth with Lifshitz scaling and hyperscaling violation, J. High Energy Phys. 07 (2018) 042.

[21] M. Alishahiha, K. B. Velni, and M. R. M. Mozaffar, Subregion action and complexity, Phys. Rev. D 99, 126016 (2019).

[22] M. Lezgi and M. Ali-Akbari, A note on holographic subregion complexity and QCD phase transition, Phys. Rev. D 101, 026022 (2020).

[23] M. asadi, On volume subregion complexity in nonconformal theories, Eur. Phys. J. C 80, 681 (2020).

[24] M. Lezgi, M. Ali-Akbari, and M. Asadi, Non-conformality, subregion complexity and meson bounding, arXiv:2011 .11625 .
[25] L. Susskind, Three lectures on complexity and black holes, arXiv:1810.11563.

[26] A. R. Brown and L. Susskind, Second law of quantum complexity, Phys. Rev. D 97, 086015 (2018).

[27] S.-J. Zhang and E. Abdalla, Holographic thermalization in charged dilaton anti-de Sitter spacetime, Nucl. Phys. B896, 569 (2015).

[28] H. Ebrahim and M. Ali-Akbari, Dynamically probing strongly-coupled field theories with critical point, Phys. Lett. B 783, 43 (2018).

[29] S. I. Finazzo, R. Rougemont, M. Zaniboni, R. Critelli, and J. Noronha, Critical behavior of non-hydrodynamic quasinormal modes in a strongly coupled plasma, J. High Energy Phys. 01 (2017) 137.

[30] S. Amiri-Sharifi, M. Ali-Akbari, and H. R. Sepangi, Electric field quench, equilibration and universal behavior, Phys. Rev. D 91, 126007 (2015).

[31] S. Amiri-Sharifi, M. Ali-Akbari, A. Kishani-Farahani, and N. Shafie, Double relaxation via AdS/CFT, Nucl. Phys. B909, 778 (2016).

[32] L. Susskind, Entanglement is not enough, Fortschr. Phys. 64, 49 (2016).

[33] H. Ebrahim, M. Asadi, and M. Ali-Akbari, Evolution of holographic complexity near critical point, J. High Energy Phys. 09 (2019) 023.

[34] B. Amrahi, M. Ali-Akbari, and M. Asadi, Holographic entanglement of purification near a critical point, Eur. Phys. J. C 80, 1152 (2020).

[35] H. Ebrahim and G. M. Nafisi, Holographic mutual information and critical exponents of the strongly coupled plasma, Phys. Rev. D 102, 106007 (2020).

[36] B. Chen, W.-M. Li, R.-Q. Yang, C.-Y. Zhang, and S.-J. Zhang, Holographic subregion complexity under a thermal quench, J. High Energy Phys. 07 (2018) 034.

[37] Y. Ling, Y. Liu, C. Niu, Y. Xiao, and C.-Y. Zhang, Holographic subregion complexity in general Vaidya geometry, J. High Energy Phys. 11 (2019) 039.

[38] Yu. T. Zhang, X.-M. Kuang, Y.-Z. Li, and J.-P. Wu, Holographic subregion complexity under a thermal quench in an Einstein-Maxwell-axion theory with momentum relaxation, Phys. Rev. D 101, 106024 (2020).

[39] R. Auzzi, G. Nardelli, F. I. S. Massolo, G. Tallarita, and N. Zenoni, On volume subregion complexity in Vaidya spacetime, J. High Energy Phys. 11 (2019) 098.

[40] D. A. Lidar and K. B. Whaley, Decoherence-free subspaces and subsystems, in Irreversible Quantum Dynamics, Springer Lecture Notes in Physics, Vol. 622, edited by F. Benatti and R. Floreanini (Springer, Berlin, 2003), pp. 83-120. 